\title{
Hereditary spastic paraplegia with recessive trait caused by mutation in KLC4 gene
}

\author{
Fatih Bayrakli ${ }^{1,2}$, Hatice Gamze Poyrazoglu ${ }^{3}$, Sirin Yuksel $^{4}$, Cengiz Yakicier ${ }^{4}$, Bekir Erguner ${ }^{5,6}$, \\ Mahmut Samil Sagiroglu ${ }^{5}$, Betul Yuceturk ${ }^{5}$, Bugra Ozer ${ }^{5,6}$, Selim Doganay ${ }^{7}$, Bahattin Tanrikulu ${ }^{8}$, Askin Seker ${ }^{1,2}$, \\ Fatih Akbulut ${ }^{1,2}$, Ali Ozen ${ }^{1,2}$, Huseyin Per ${ }^{9}$, Sefer Kumandas ${ }^{9}$, Yasemin Altuner Torun ${ }^{10}$, Yasar Bayri ${ }^{1,2}$, \\ Mustafa Sakar ${ }^{1,2}$, Adnan Dagcinar ${ }^{1,2}$ and Ibrahim Ziyal ${ }^{1,2}$
}

We report an association between a new causative gene and spastic paraplegia, which is a genetically heterogeneous disorder. Clinical phenotyping of one consanguineous family followed by combined homozygosity mapping and whole-exome sequencing analysis. Three patients from the same family shared common features of progressive complicated spastic paraplegia. They shared a single homozygous stretch area on chromosome 6 . Whole-exome sequencing revealed a homozygous mutation (c.853_871del19) in the gene coding the kinesin light chain 4 protein (KLC4). Meanwhile, the unaffected parents and two siblings were heterozygous and one sibling was homozygous wild type. The 19 bp deletion in exon 6 generates a stop codon and thus a truncated messenger RNA and protein. The association of a KLC4 mutation with spastic paraplegia identifies a new locus for the disease.

Journal of Human Genetics (2015) 60, 763-768; doi:10.1038/jhg.2015.109; published online 1 October 2015

\section{INTRODUCTION}

Spastic paraplegia (SP) in hereditary form is a genetically and clinically heterogeneous group of diseases with distinctive features of axonal degeneration in corticospinal tracts. Progressive spasticity and weakness of the lower limbs are hallmarks of the disease, and in complicated subtypes, additional neurological and systemic manifestations may be present. $^{1}$

SP can be classified as autosomal dominant, autosomal recessive, $\mathrm{X}$-linked and mitochondrial according to the inheritance pattern. Many causative genes as for autosomal recessive SP have been reported. Considering these genes, there are several pathogenic mechanisms underlying the SP, including oxidative stress, axonal transport dysfunction, abnormal lipid metabolism, abnormal DNA repair, myelination dysregulation, autophagy, axon development defects, endosome membrane trafficking and vesicle formation defects, abnormal cellular signaling in protein morphogenesis, and abnormal membrane traffic and organelle shaping. ${ }^{1}$

We report on a novel association of the kinesin light chain 4 protein (KLC4) gene with complicated autosomal recessive SP.

\section{MATERIALS AND METHODS}

The study was approved by the Marmara University Ethic Committee of Clinical Research (protocol number: 09.2014.0312/70737436-050.06.04-). We identified a family in eastern Turkey diagnosed with a progressive neurodegenerative disease based on clinical, radiological records and biochemical laboratory results. After obtaining consent, blood samples were obtained. DNA was then prepared using standard techniques.

\section{Exome sequencing and homozygosity mapping}

Genomic DNA samples of III-1, III-2, IV-1, IV-5 and IV-6 were prepared for whole-exome sequencing using a TruSeq Sample Preparation Kit (Illumina, San Diego, CA, USA). Exonic regions were captured using Nimblegen SeqCap EZ (Roche, Basel, Switzerland). A TruSeq PE Cluster Kit v3-cBot-HS was used for paired-end cluster generation and a TruSeq SBS Kit v3-HS reagent kit for sequencing post-capture libraries (Illumina). Initial clustering was performed on an Illumina cBot machine and paired-end sequencing on an Illumina HiSeq 2500 system with a read length of PE $2 \times 104$. All the procedures were done according to manufacturer's instructions. Base calling and image analysis was done using Illumina's Real Time Analysis software version 1.13 with default parameters.

The paired-end sequence reads were aligned to the human genome (hg19) using BWA software. ${ }^{2}$ SAMtools was used to remove PCR duplicates. ${ }^{3}$ After duplication removal, targeted exome regions depth-of-coverage was calculated using BEDtools. ${ }^{4}$ Realignment around insertions and deletions (indels) was performed using The Genome Analysis Toolkit v1.6 (GATK) IndelRealigner. ${ }^{5}$ To discover single-nucleotide substitutions and small indels, the GATK Unified Genotyper was run on aligned reads with recommended parameters. Variants

${ }^{1}$ Department of Neurosurgery, Marmara University School of Medicine, Istanbul, Turkey; ${ }^{2}$ Institute of Neurological Sciences, Marmara University, Istanbul, Turkey; ${ }^{3}$ Department of Child Neurology, Firat University School of Medicine, Elazig, Turkey; ${ }^{4}$ Department of Medical Biology, Acibadem University School of Medicine, Istanbul, Turkey; ${ }^{5}$ Advanced Genomics and Bioinformatics Research Center (IGBAM), BILGEM, The Scientific and Technological Research Council of Turkey (TUBITAK), Kocaeli, Turkey; ${ }^{6}$ Department of Molecular Biology Genetics and Bioengineering, Sabanci University Faculty of Engineering and Natural Sciences, Istanbul, Turkey; ${ }^{7}$ Department of Pediatric Radiology, Erciyes University Children's Hospital, Kayseri, Turkey; ${ }^{8}$ Neurosurgery Clinic, Marmara University Pendik Research and Training Hospital, Istanbul, Turkey; ${ }^{9}$ Department of Child Neurology, Erciyes University School of Medicine, Kayseri, Turkey and ${ }^{10}$ Child Health and Diseases Clinic, Kayseri Education and Research Hospital, Kayseri, Turkey Correspondence: Dr F Bayrakli, Department of Neurosurgery, Institute of Neurological Sciences, Marmara University School of Medicine, Istanbul 34720, Turkey. E-mail: fatih.bayrakli@marmara.edu.tr

Received 22 May 2015; revised 15 August 2015; accepted 17 August 2015; published online 1 October 2015 
Table 1 Primer used in sequencing experiments for genomic DNA and cDNA

\begin{tabular}{ll}
\hline Primer name & Oligonucleotide chain \\
\hline KLC4 exon 6 forward for genomic DNA & CCAGGCTTCCTGTCTCCT \\
KLC4 exon 6 reverse for genomic DNA & CTTATGCCTCCTTCCTCAGC \\
KLC4 exon 5 forward for cDNA & GGTGGATATGAGATCCCAGCA \\
KLC4 exon 6 reverse for cDNA mutation site & TCAGCAGGTGGGCTGGTC \\
\hline
\end{tabular}

Abbreviations: cDNA, complementary DNA; KLC4, kinesin light chain 4 protein.

with a quality score lower than 50 were discarded. The remaining variants were annotated for novelty by comparing with dbSNP (build 135).

Functional annotation of variants with an adequate quality score was done using SnpEff and SnpSift. ${ }^{6}$ Variants of interest were then sifted using VarSifter ${ }^{7}$ in which positions that indicated a homozygous variant in affected siblings, and a heterozygous variant in the mother and father were selected for further inspection. HomSI ${ }^{8}$ was used for homozygosity mapping analysis. Variants that fit the segregation pattern and which also existed inside the homozygous stretch region were selected as the highest-ranking candidates. The final set of selected variants was visually inspected using Integrative Genomics Viewer. ${ }^{9}$

\section{Mutation confirmation by Sanger sequencing}

To confirm the mutation, PCR primers covering the mutation site in KLC4 were designed using PRIMER3 (http://bioinfo.ut.ee/primer3-0.4.0/ website; Table 1). BLAST homology analysis was performed using the National Center for Biotechnology Information Web tool (http://www.ncbi.nlm.nih.gov/blast website) to compare individual sequences with wild-type sequences. Mutation harboring exon was amplified using standard PCR techniques and was directly sequenced for each family member.

\section{RNA isolation, cDNA synthesis and cDNA sequencing}

Total RNA was isolated from EDTA peripheral blood samples by QIAamp RNA Blood Mini Kit (Qiagen, Valencia, CA, USA) according to manufacturer's instructions. Complementary DNA (cDNA) was synthesized using $1 \mu \mathrm{g}$ of RNA using random primers (Promega, Madison, WI, USA) and an Omniscript Reverse Transcription Kit (Qiagen).

PRIMER3 (http://bioinfo.ut.ee/primer3-0.4.0/ website) was used for primer design (Table 1). The reverse primer was designed to be specific for the mutant allele, and to not amplify a normal allele. Reverse transcriptase PCR amplicons were used as templates for direct Sanger DNA sequencing using the same primers for PCR amplification.

\section{RESULTS}

\section{Patient phenotypes}

Three affected and three clinically healthy children and their parents in a consanguineous marriage (Figure 1A).

Because the disease in our family is progressive in nature, we will start to present the clinical and neurological examination features in detail from the youngest through the eldest affected children. Patient IV-6 is an 8-year-old girl presented to our child neurology department with the complaints of deterioration in walking, decrease in vision and hearing over time. She was born to term vaginal delivery, cried immediately after birth without cyanosis. The patient had started independent walking while she was 13 months old and her development was normal until the age of 3 years. After this point, the patient had started to experience a decrease in hearing and vision and deterioration in walking. She can partially understand what is said loudly, can obey simple commands and has retardation in mental capacity. She has an ataxic gait and can barely walk short distance, and cannot walk long distance and hurdle. Her eye movements are normal, has isochoric pupils with bilateral diminished light reflexes and horizontal nystagmus. Deep tendon reflexes of the patient were hyperactive which was more prominent in the lower extremities, had positive Babinski reflex, positive clonus sign and mildly increased tonus in the bilateral lower extremities. Muscle strengths were 4/5 in the lower extremities, whereas they were normal in the upper extremities and there were no muscle atrophies. Skillful hand movements were absent in bilateral hands. There was no facial dysmorphic features, bilateral cubitis valgus was present bilaterally in upper extremities; there was no contracture, and mild internal deviation was seen in the left foot (Figure 1B). Owing to very limited cooperation, sensory system examination was not optimal but the patient was pulling her upper and lower extremities after painful stimulus created by needle. Routine biochemical tests, blood ammonia and pyruvate levels, quantitative plasma amino acid analysis, tandem MS analysis, urine amino acid and organic acid analysis, cerebrospinal fluid amino acids and lactate levels were in the normal range. Blood lactate level was higher than the normal range $\left(30 \mathrm{mg} \mathrm{dl}^{-1}\right.$, normal: $4.5-19.8 \mathrm{mg} \mathrm{dl}^{-1}$ ). Blood very-long-chain lipid acid and lysosomal enzyme levels were normal. Electromyography and nerve conduction studies revealed mild peroneal nerve demyelinating polyneuropathy and slowing of the nerve conduction velocities. Eye examination of the patient showed bilateral pale optic disks and retinitis pigmentosa. Bilateral auditory brainstem response test diagnosed near total sensorineural type hearing loss. The patient's IQ level was mildly retardate with the score of 65 . Cranial magnetic resonance imaging (MRI) of the patient revealed signal changes bilaterally in the dentate nucleus of cerebellum, posterior leg of internal capsule and the subcortical white matter (Figure 1C). Spinal MRI of the patient radiologically was normal.

Patient IV-5 is a 12-year-old girl presented to our child neurology department with the complaints of inability to walk without assistance, decrease in vision and hearing over time. She was born to term vaginal delivery, cried immediately after birth without cyanosis. The patient had started independent walking while she was 12 months old and her development was normal until the age of 3 years. After this point, the patient had started to experience a decrease in hearing and vision and deterioration in walking. She is conscious, has no awareness and reaction to sounds. She can sit without assistance but cannot stand or walk without assistance. Her eye movements are normal, has isochoric pupils with bilateral diminished light reflexes and horizontal nystagmus. Deep tendon reflexes of the patient were hyperactive, which was more prominent in lower extremities, had positive Babinski reflex, positive clonus sign and severe spasticity in the bilateral lower extremities. Muscle strengths were $3 / 5$ in the lower extremities, whereas they were normal in the upper extremities; and there were prominent muscle atrophies in the lower extremities and crosses both lower extremities. Skillful hand movements were absent in bilateral hands. There was no facial dysmorphic features. Bilateral cubitis valgus was present bilaterally in the upper extremities, there were no contractures and prominent internal deviation was seen in the left foot (Figure 1B). Any reaction was obtained to painful stimulus, such as pulling the leg or arm. Routine biochemical tests, blood ammonia and pyruvate levels, quantitative plasma amino acid analysis, tandem MS analysis, urine amino acid and organic acid analysis, cerebrospinal fluid amino acids and lactate levels were in the normal range. Blood lactate level was higher than the normal range ( $29 \mathrm{mg} \mathrm{dl}^{-1}$, normal: $4.5-19.8 \mathrm{mg} \mathrm{dl}^{-1}$ ). Blood very-long-chain lipid acid and lysosomal enzyme levels were normal. Electromyography and nerve conduction studies revealed demyelinating polyneuropathy as mild in upper extremities and as 
A

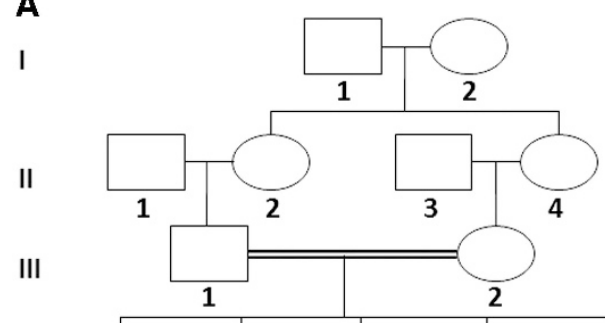

C

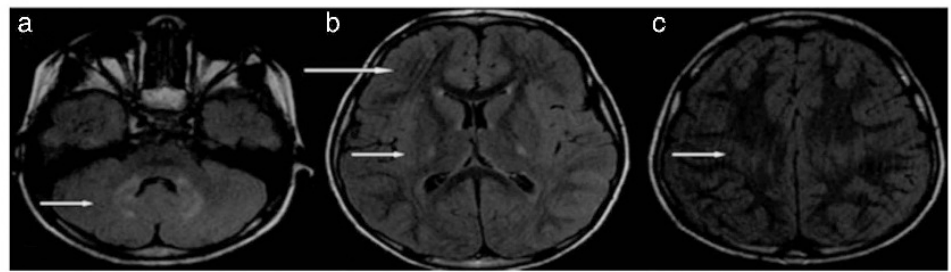

IV

1

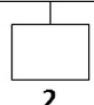

2

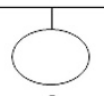

4

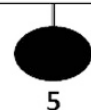

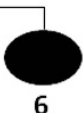

B
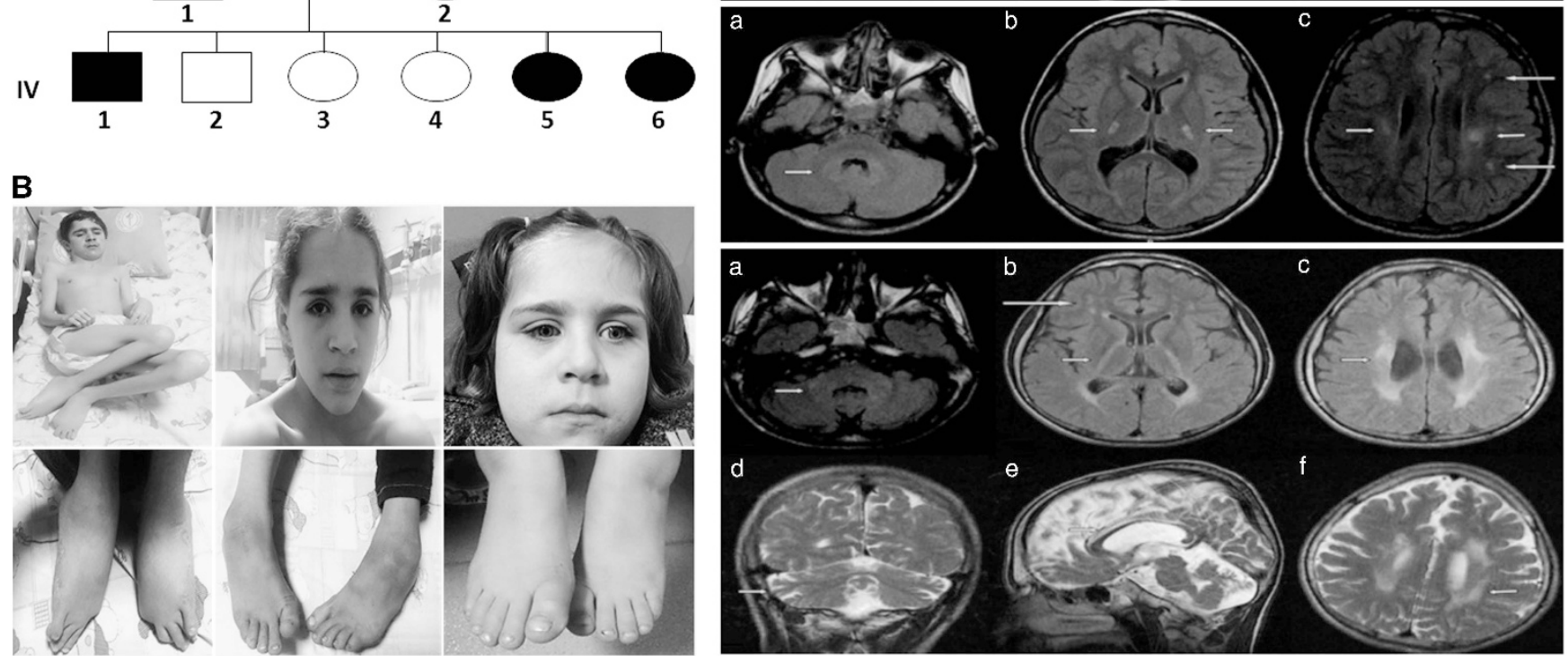

Figure 1 (A) Family pedigree. (B) Photographs of patients that have no facial dysmorphic features: left column (patient IV-1), extremity contractures and foot deformity is prominent; middle column (patient IV-5), internal deviation of the foot is prominent; right column (patient IV-6) shows mild foot deformities. (C) Cranial magnetic resonance imaging (MRI) of the patients. Upper row (patient IV-6): axial FLAIR images show diffuse increased signals within dentate nucleus (arrow; a), increased signals in the bilateral corticospinal tracts in the posterior legs of internal capsule extending to periventricular white matter in centrum semiovale (short arrows; $\mathbf{b}$ and $\mathbf{c}$ ) and foci of abnormal signals in the subcortical white matter (Iong arrow; b). Middle row (patient IV-5): axial FLAIR images show diffuse very mild increased signals within dentate nucleus (arrow; a), increased signals in the bilateral corticospinal tracts in the posterior legs of internal capsule extending to periventricular white matter in centrum semiovale (short arrows; $\mathbf{b}$ and $\mathbf{c}$ ) and foci of abnormal signals in the subcortical white matter (long arrows; c). Lower row (patient IV-1): axial FLAIR images show diffuse very mild increased signals within dentate nucleus (arrow; a), increased signals in the bilateral corticospinal tracts in the posterior legs of internal capsule extending to periventricular white matter in centrum semiovale (short arrows; $\mathbf{b}$ and $\mathbf{c}$ ) and foci of abnormal signals in the subcortical white matter (long arrows; b). Coronal T2-weighted image shows mild cerebellar atrophy (arrow; d), sagittal T2-weighted image shows thin body of corpus callosum (arrow; e) and axial T2-weighted image shows increased T2 signals in the centrum semiovale (arrow) and mild cerebral atrophy (f). A full color version of this figure is available at the Journal of Human Genetics journal online.

severe in lower extremities and slowing of the nerve conduction velocities. Eye examination of the patient showed retinitis pigmentosa. She had moderate to severe sensorineural type hearing loss. Patient's IQ test score was 40-50. Cranial MRI showed minimal increased signal in the dentate nucleus of cerebellum, but posterior leg of internal capsule and subcortical white matter signal changes were prominent (Figure 1C). Spinal MRI of the patient radiologically was normal.

Patient IV-1 is a 19-year-old boy presented to our child neurology department with the complaints of inability to walk, blindness and deafness. He was born to term vaginal delivery, cried immediately after birth without cyanosis. The patient had started independent walking while he was 12 months old and his development was normal until the age of 3 years. After this point, the patient had started to experience progressive loss of hearing, vision and deterioration in walking. He lost his walking ability while he was 12 years old and became blind and deaf 5 years ago. He is conscious, has no awareness, no reaction to sounds and makes incomprehensible sounds. His eye movements are normal, has isochoric pupils with negative bilateral light reflexes and positive horizontal nystagmus. Deep tendon reflexes were absent and prominent muscle atrophy was seen in both upper and lower extremities. There were flexion contractures in elbow, knee and ankle joints and deformities in hand and foot fingers. Muscle strengths were $2 / 5$ in the lower extremities and $3 / 5$ in the upper extremities. He crosses both lower extremities. There was no facial dysmorphic features (Figure 1B). Any reaction was obtained to painful stimulus such as pulling the leg or arm. Routine biochemical tests, blood ammonia and pyruvate levels, quantitative plasma amino acid analysis, tandem MS analysis, urine amino acid and organic acid analysis, cerebrospinal fluid amino acids and lactate levels were in the normal range. Blood lactate level was higher than the normal range (30 mg dl${ }^{-1}$, normal: $4.5-19.8 \mathrm{mg} \mathrm{dl}^{-1}$ ). Blood very-long-chain lipid acid and lysosomal enzyme levels were normal. Electromyography and nerve conduction studies revealed demyelinating polyneuropathy as mild in the upper extremities and as severe in the lower extremities and slowing of the nerve conduction velocities. Eye examination of the patient showed retinitis pigmentosa bilaterally. She had bilateral severe sensorineural type hearing loss. The patient's IQ test score was 25-30. Cranial MRI features of the patient were similar to other kids but subcortical white matte changes were more striking 


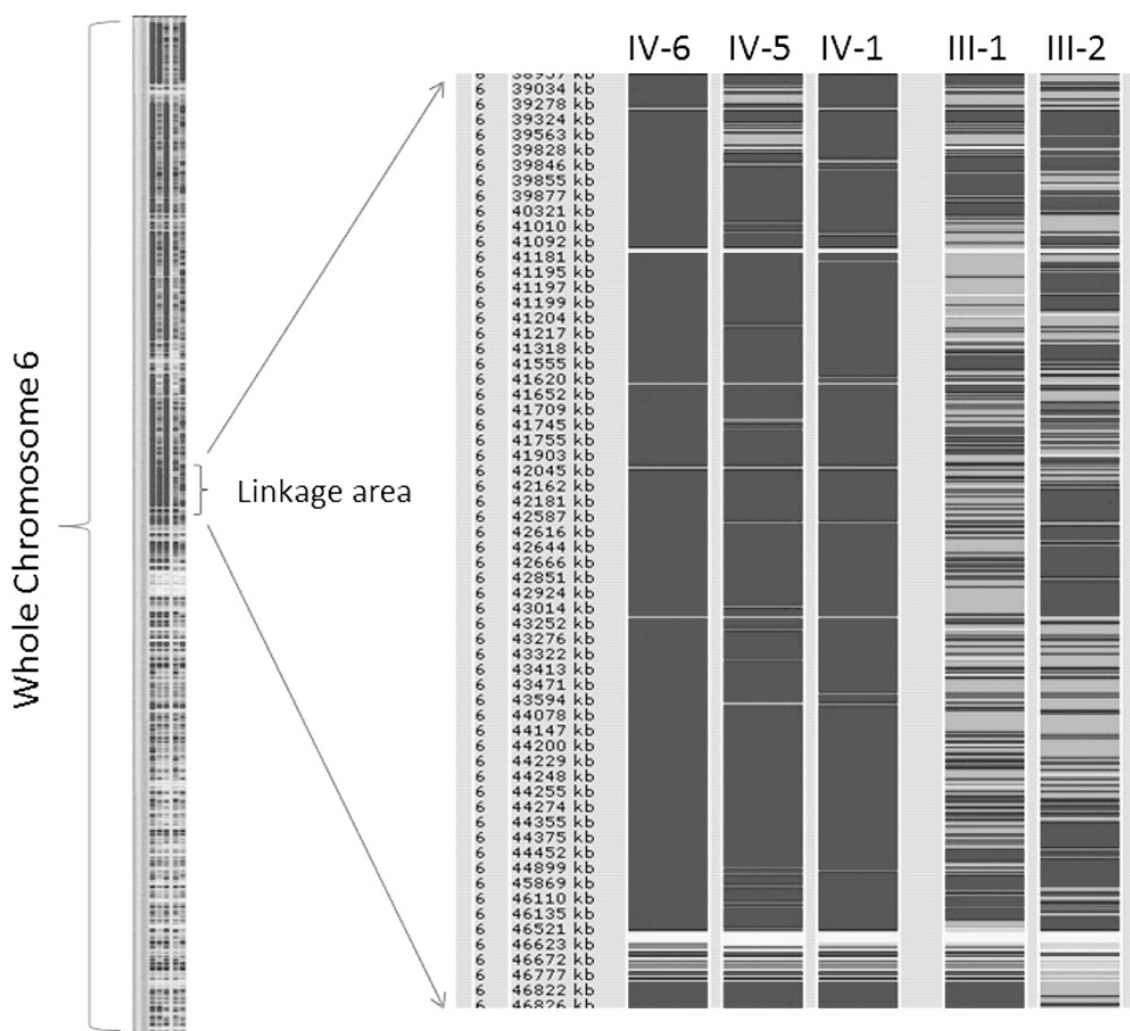

Figure 2 Homozygosity map of chromosome 6 (left) shared blue homozygous region by affected children and zoomed in style of the same region on the right. A full color version of this figure is available at the Journal of Human Genetics journal online.

than others (Figure 1C). Spinal MRI of the patient radiologically was normal.

Exome sequencing, homozygosity mapping and Sanger sequencing Genomic DNA samples from family members III-1, III-2, IV-1, IV-5 and IV-6 were analyzed by whole-exome sequencing for causative mutations that segregated with the pedigree status. Mean coverage of $\times 44$ was achieved, and $94 \%$ of all targeted bases were read more than four times, which was sufficient to identify novel homozygous variants with high specificity. Homozygosity mapping analysis showed a shared homozygous stretch region in the three affected children in chromosome 6, while the parents were heterozygous between 40 and $46 \mathrm{Mb}$ of the chromosome (Figure 2). A mutation in the KLC4 gene was identified within this homozygous stretch area that segregated with pedigree status (Supplementary Table 1). The mutation was c.853_871del19, and caused stop codon formation at 277 th aa, which is also located in a highly conserved region between species. Furthermore, we could not find this variation in our own in-house exomes $(n=650)$ from individuals with unrelated diseases and on data bases of normal controls such as 1000 Genomes (http://www.1000genomes.org/home), human genetic variation data base (http://www.genome.med.kyoto-u.ac.jp/SnpDB/) and Exome Variant Server (http://evs.gs.washington.edu/EVS/). Sanger sequencing of the mutation containing exon 6 of the KLC4 gene confirmed the exome sequencing result (Figure 3a). This mutation destroys half of the KLC4 gene protein including tetratricopeptide repeat domains and probably affects the cargo binding function of the protein (Figure $3 \mathrm{~b}$ ).

\section{RNA study}

As we applied our reverse primer to the deletion site, cDNA PCR was positive for affected and carrier family members and negative for a homozygous wild-type healthy child and healthy control (Figure 4a). This also proved the existence of deletion mutation on RNA. Sequencing of the cDNA PCR product confirmed the result (Figure 4b).

\section{DISCUSSION}

Our study presents for the first time proof that a mutation in the KLC4 gene is related to SP. The clinical phenotype shows similarities with the phenotype of mice and drosophila knockout models of the KLC1 gene, suggesting that the KLC4 protein is also related to neuronal transport. Radiological findings add further evidence of this inference.

Kinesins have diverse functions, including the transport of cargo and regulation of microtubule dynamics. As such, microtubule-based transport defects are deleterious to neuronal activity. ${ }^{10}$ Various kinesin proteins have been reported with structures having two KHCs and two KLCs. ${ }^{11}$ Four isoforms of KLC exist in humans: KLC1, KLC2, KLC3 and KLC4. ${ }^{10}$ Little is known about the KLC4 gene function. Databases indicate that the KLC4 gene is located on chromosome 6p21.1 and has multiple isoforms.

The KLC contains three domains: the N-terminal coiled-coil domain (heptad repeat)/Rab-5 bind that binds to the KHC, a tetratricopeptide repeat and the C-terminal domain. The latter two domains of the KLC are primarily involved in the binding of cargo, functioning as a physical linker between $\mathrm{KHC}$ and cargo. ${ }^{10,12}$ Our mutation in the KLC4 gene placed a stop codon at the 277 amino acid position, such that most of the tetratricopeptide repeat and the entire 
a

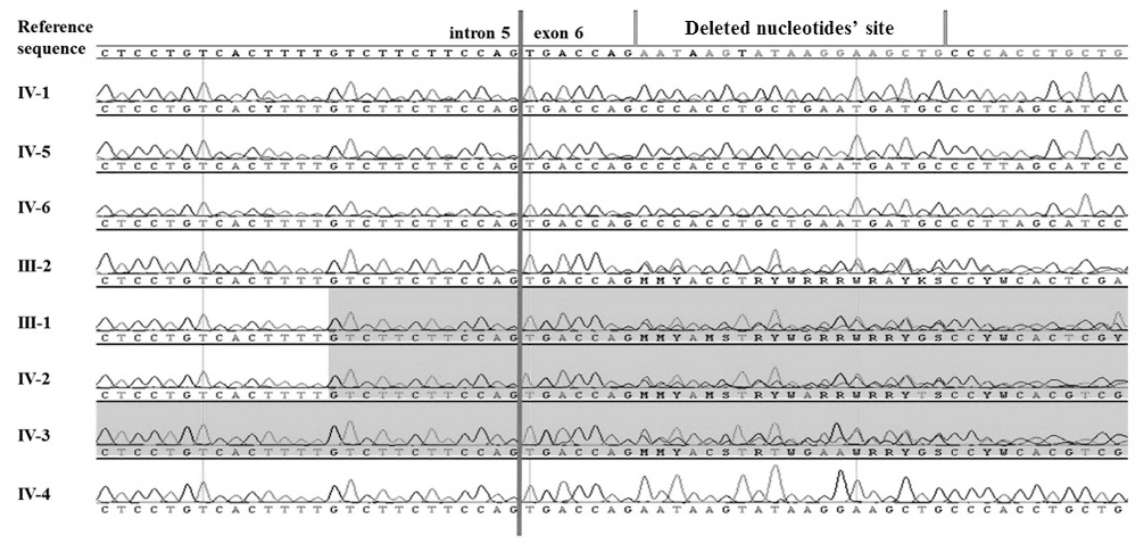

b

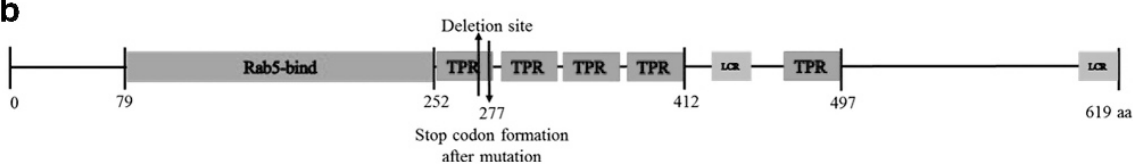

Figure 3 (a) Sanger sequencing results of all family members, vertical red lines indicate mutation site; (b) KLC4 protein domains and mutation site. LCR, low complexity region; TPR, tetratricopeptide region. A full color version of this figure is available at the Journal of Human Genetics journal online.

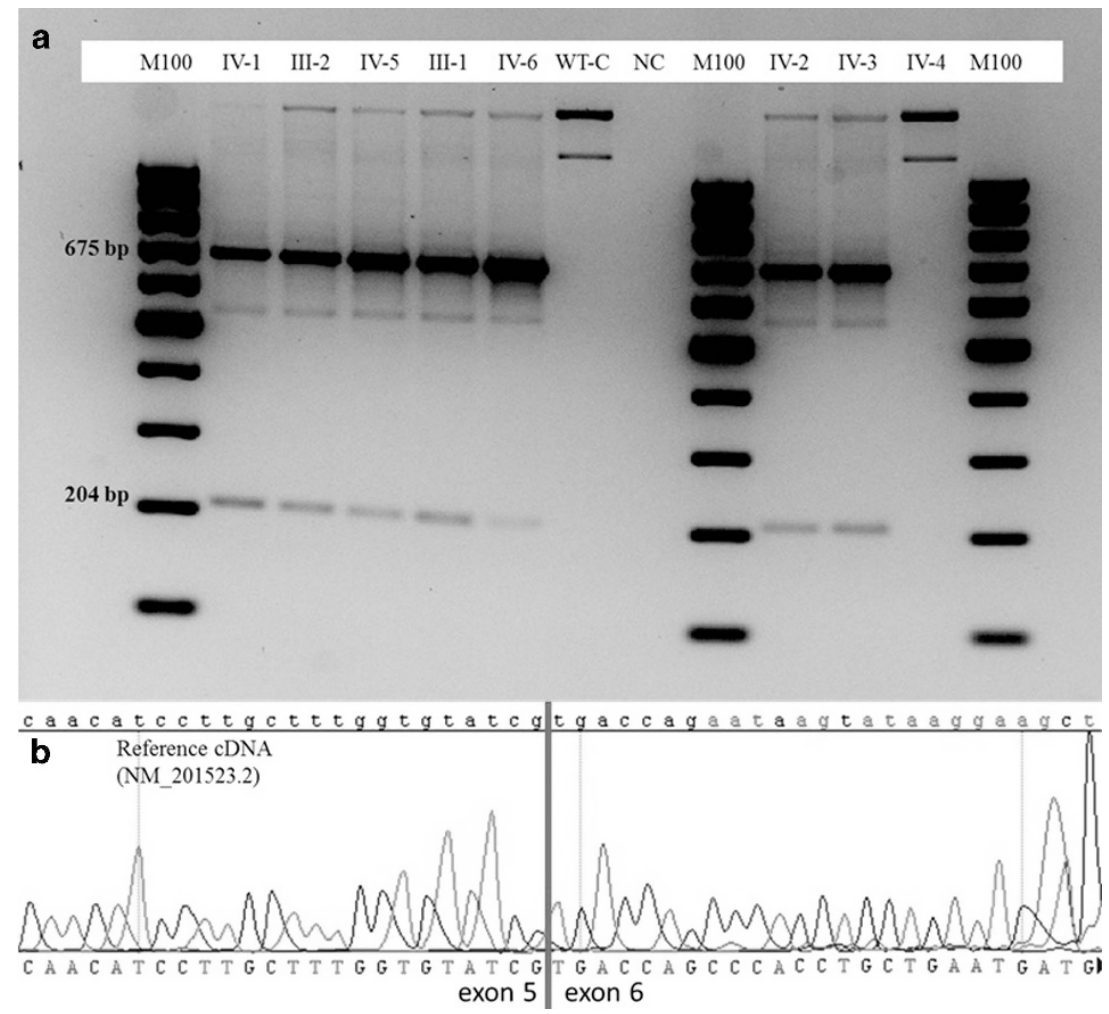

Figure 4 (a) cDNA PCR gel view, because reverse primer used were placed to mutation site, band formation was seen in homozygous mutant patients (IV-1, IV-5 and IV-6) and carriers (III-1, III-2, IV-2 and IV3), but we cannot see band formation in homozygous wild-type healthy child (IV-4) and WT-C at the level of 204 bp. M100, 100 bp ladder; NC, negative control; WT-C, wild-type control. (b) cDNA sequencing result of homozygous mutation. A full color version of this figure is available at the Journal of Human Genetics journal online.

C-terminal domain of the KLC4 protein cannot be produced by affected patients during protein synthesis. This results in an inability to transport cargo that needs the KLC4 as a transporter because the cargo binding site of the kinesin molecule is destroyed. Thus, radiological and clinical disease emerges. These cargos are currently unknown and elucidation is left for further studies. The clinical and radiological data from our family indicates that such cargos are related to neuronal function and motor functions. 
Previous functional studies on KLC were mostly aimed at the KLC1 subtype. Gindhart et al. ${ }^{13}$ reported the phenotype of a KLC mutant Drosophila where loss of KLC function causes progressive lethargy, crawling defects and paralysis. Progressive paralysis associated with KLC function starts at the posterior end of the larva and proceeds anteriorly. In the early years of their lives, our patients' motor functions appeared normal, but after the age of three walking began to deteriorate and paralysis started in the lower extremities and progressed to the upper extremities.

Rahman et al. ${ }^{14}$ generated mutant mice lacking normal KLC1 to investigate KLC functions and concluded that mice lacking wild-type $\mathrm{KLC} 1$ are abnormal in terms of motor functions and body size. Motor function problems were also obvious in our KLC4 homozygous mutant family members.

In conclusion, our study demonstrated for the first time an association between the KLC4 gene and a human neurological disease.

\section{CONFLICT OF INTEREST}

The authors declare no conflict of interest.

\section{ACKNOWLEDGEMENTS}

Exome sequencing experiments of this study supported by The Republic of Turkey Ministry of Development Infrastructure Grant (no: 2011K120020) and BILGEM-TUBITAK (The Scientific and Technological Research Council of Turkey) (grant no: T439000)
1 Lo Giudice, T., Lombardi, F., Santorelli, F. M., Kawarai, T. \& Orlacchio, A. Hereditary spastic paraplegia: clinical-genetic characteristics and evolving molecular mechanisms. Exp. Neurol. 261, 518-539 (2014).

$2 \mathrm{Li}$, H. \& Durbin, R. Fast and accurate short read alignment with Burrows-Wheeler transform. Bioinformatics 25, 1754-1760 (2009).

3 Li, H., Handsaker, B., Wysoker, A., Fennell, T., Ruan, J., Homer, N. et al. The Sequence Alignment/Map format and SAMtools. Bioinformatics 25, 2078-2079 (2009)

4 Quinlan, A. R. \& Hall, I. M. BEDTools: a flexible suite of utilities for comparing genomic features. Bioinformatics 26, 841-842 (2010).

5 DePristo, M. A., Banks, E., Poplin, R., Garimella, K. V., Maguire, J. R., Hartl, C. et al. A framework for variation discovery and genotyping using next-generation DNA sequencing data. Nat. Genet. 43, 491-498 (2011).

6 Cingolani, P., Platts, A., Wang le, L., Coon, M., Nguyen, T., Wang, L. et al. A program for annotating and predicting the effects of single nucleotide polymorphisms, SnpEff: SNPs in the genome of Drosophila melanogaster strain w1118; iso-2; iso-3. Fly 6, 80-92 (2012).

7 Teer, J. K., Green, E. D., Mullikin, J. C. \& Biesecker, L. G. VarSifter: visualizing and analyzing exome-scale sequence variation data on a desktop computer. Bioinformatics 28, 599-600 (2012).

8 Gormez, Z., Bakir-Gungor, B. \& Sagiroglu, M. S. HomSI: a homozygous stretch identifier from next-generation sequencing data. Bioinformatics 30, 445-447 (2014).

9 Robinson, J. T., Thorvaldsdottir, H., Winckler, W., Guttman, M., Lander, E. S., Getz, G. et al. Integrative genomics viewer. Nat. Biotechnol. 29, 24-26 (2011).

10 Zhu, H., Lee, H. Y., Tong, Y., Hong, B. S., Kim, K. P., Shen, Y. et al. Crystal structures of the tetratricopeptide repeat domains of kinesin light chains: insight into cargo recognition mechanisms. PLOS ONE 7, e33943 (2012).

11 Endow, S. A., Kull, F. J. \& Liu, H. Kinesins at a glance. J. Cell Sci. 123, 3420-3424 (2010).

12 Kamal, A. \& Goldstein, L. S. Principles of cargo attachment to cytoplasmic moto proteins. Curr. Opin. Cell Biol. 14, 63-68 (2002).

13 Gindhart, J. G. Jr., Desai, C. J., Beushausen, S., Zinn, K. \& Goldstein, L. S. Kinesin light chains are essential for axonal transport in Drosophila. J. Cell Biol. 141, 443-454 (1998).

14 Rahman, A., Kamal, A., Roberts, E. A. \& Goldstein, L. S. Defective kinesin heavy chain behavior in mouse kinesin light chain mutants. J. Cell Biol. 146, 1277-1288 (1999).

Supplementary Information accompanies the paper on Journal of Human Genetics website (http://www.nature.com/jhg) 